\title{
Production of a chromium Bose-Einstein condensate
}

\author{
Axel Griesmaier, Jürgen Stuhler, Tilman Pfau \\ 5. Physikalisches Institut, Universität Stuttgart, \\ Pfaffenwaldring 57, 70550 Stuttgart, Germany
}

\begin{abstract}
The recent achievement of Bose-Einstein condensation of chromium atoms [1] has opened longedfor experimental access to a degenerate quantum gas with long-range and anisotropic interaction. Due to the large magnetic moment of chromium atoms of $6 \mu \mathrm{B}$, in contrast to other Bose-Einstein condensates (BECs), magnetic dipole-dipole interaction plays an important role in a chromium BEC. Many new physical properties of degenerate gases arising from these magnetic forces have been predicted in the past and can now be studied experimentally. Besides these phenomena, the large dipole moment leads to a breakdown of standard methods for the creation of a chromium BEC. Cooling and trapping methods had to be adapted to the special electronic structure of chromium to reach the regime of quantum degeneracy. Some of them apply generally to gases with large dipolar forces. We present here a detailed discussion of the experimental techniques which are used to create a chromium BEC and allow us to produce pure condensates with up to $10^{5}$ atoms in an optical dipole trap. We also describe the methods used to determine the trapping parameters.
\end{abstract}

\section{INTRODUCTION}

Among the species that have been used to create degenerate quantum gases, chromium is outstanding with respect to its very large magnetic dipole moment of $6 \mu_{B}$ (where $\mu_{B}$ is Bohr's magneton) in the ground state ${ }^{7} S_{3}$. This dipole moment leads to a magnetic dipole-dipole interaction (MDDI) which has a strength comparable to the interaction arising from the isotropic and effectively short range interatomic (contact) potential. Since the MDDI scales with the square of the magnetic moment, it is a factor of 36 stronger for chromium than for alkali atoms. Although atomic Bose-Einstein condensates are very dilute systems, strength, range and symmetry of the weak interactions between the atoms determine the essential properties of degenerate quantum gases and give rise to many phenomena that can be observed.

In the Bose-Einstein condensates that have been produced so far [2, 3, 4, 5, 6, 7, 8, 9], interaction between the atoms is governed by the contact potential. Many exciting effects of these interactions have been studied (for an overview see e.g. [10, 11]) and are subject to still growing interest of both, theorists and experimentalists. Recently, BECs with contact interaction have been used as model systems for solid-state physics problems like the Mott-metal-insulator transition 12, 13]. Tuneable contact interactions have been used to realize new states of quantum matter like a Tonks-Girardeau gas [14] and to produce molecular Bose-Einstein condensates 15, 16. The crossover from a molecular BEC to a degenerate Fermi gas has been studied [17, 18, 19, 20] and superfluidity and vortices have been observed in a strongly interacting Fermi gas 21]. In contrast, the MDDI between chromium atoms is long-range and anisotropic. The presence of an interaction with different symmetry and range leads to new properties of such a gas. A change in the aspect ratio of the condensate after ballistic expansion of a dipolar condensate has been predicted [22] and could be observed very recently [23].
If one of the Feshbach-resonances of chromium 24] is used to tune the contact-interaction close to zero, the MDDI can even become the dominant interaction. For such a regime, new kinds of quantum phase transitions 25 have been predicted. The ground state and stability [26, 27, 28] of a dipolar BEC can also be studied. In a pancake-like trapping geometry, the occurence of a Maxon-Roton in the excitation spectrum has been predicted [29]. Furthermore the MDDI is also tuneable [30] which allows for studies of a degenerate quantum gas in different regimes where atoms are interacting dominantly via either short-range and isotropic or long-range and anisotropic potentials.

\section{BOSE-EINSTEIN CONDENSATION OF ${ }^{52} \mathrm{CR}$}

Bose-Einstein condensation of chromium is achieved in a crossed optical dipole trap using the methods described in section III of this paper where a schematic illustration of the setup can also be found. We identify the presence of a Bose-Einstein condensate by anisotropic expansion when the condensate is released from an anisotropic trap with a tighter confinement in $\mathrm{x}$ and $y$-direction and a less tight confinement $\mathrm{z}$-direction. Figure 1 shows time of flight images of such a condensate taken after variable time of ballistic expansion between $2 \mathrm{~ms}$ and $16 \mathrm{~ms}$. The appearance of a two-component distribution in the density profile of the cloud also signalizes the presence of a condensed fraction. The point of emerging degeneracy becomes obvious when the number of atoms in the condensate and the thermal fraction are determined separately for different final laser powers. These numbers can be obtained by fitting a two-dimensional two-component distribution function to the density profiles of the cloud. The inset of fig. 2) shows a cut through the center of such a distribution with a line displaying the result of a two-component 


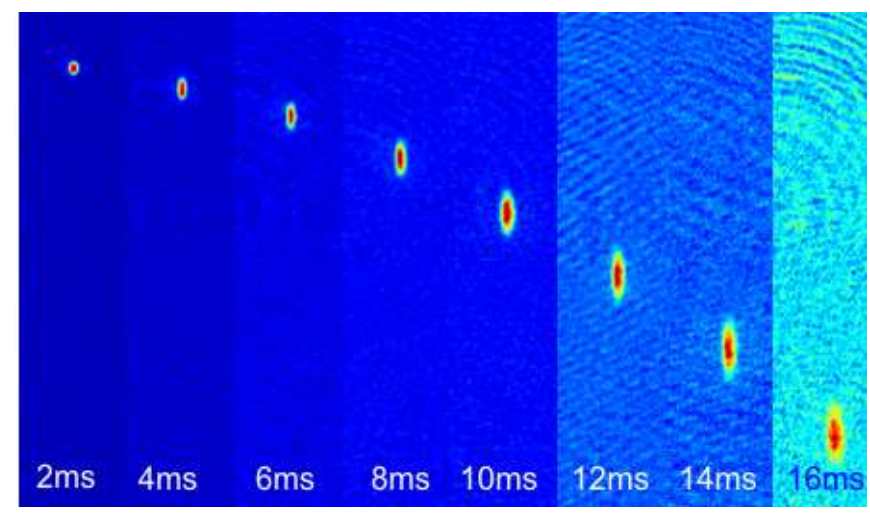

FIG. 1: Time of flight absorption images of an expanded almost pure condensate of about 40.000 atoms after expansion times between $2 \mathrm{~ms}$ and $16 \mathrm{~ms}$. The pictures are in the $\mathrm{y}-\mathrm{z}$ plane of our setup, $\mathrm{z}$ being the horizontal direction.

fit. If the trapping potential is well known, not only the number of atoms in the thermal cloud but also the temperature of the cloud can be calculated from the width of the Gaussian. We have precisely measured the trap frequencies in all directions and at different laser powers using the methods described in section IV This calibration allows us to calculate temperatures, densities and phase space densities from one time-of-flight picture. The temperatures obtained with this method have been compared to the results of much more precise timeof-flight series and are concordant with these results within a maximum deviation of $8 \%$ if the time of flight is not too short. The determination of the number of atoms and widths vastly depends on the quality of the images. To reduce fringe patterns usually present in absorption images due to fluctuations of laser power and intensity or mechanical vibrations between the separate images, we have implemented a method developed by the Sengstock group 31] in our image evaluation software. This method allows the later reduction of fringes without loss of information. This way, we where able to improve our previous analysis [1] of the dependence of the fraction of condensed atoms $\left(N_{0} / N\right)$ on the ratio of the temperature of the cloud to the critical temperature $\left(T / T_{C}\right)$. The corresponding data are shown as open circles in fig. 2 When we approach the critical temperature from above $\left(T / T_{C}>1\right)$, a kink in the condensate fraction plot marks the onset of Bose-Einstein condensation and provides an experimental value for the critical temperature of $T_{\text {exp }} \sim 700 \mathrm{nK}$. Based on the trap frequencies, number of atoms and temperature, we have also calculated the expected condensate fraction when finite size effects as well as a correction arising from the contact interaction [32] are taken into account. These expected values are represented by black dots in figure 2 and demonstrate a very good agreement of our data with the predicted dependence.

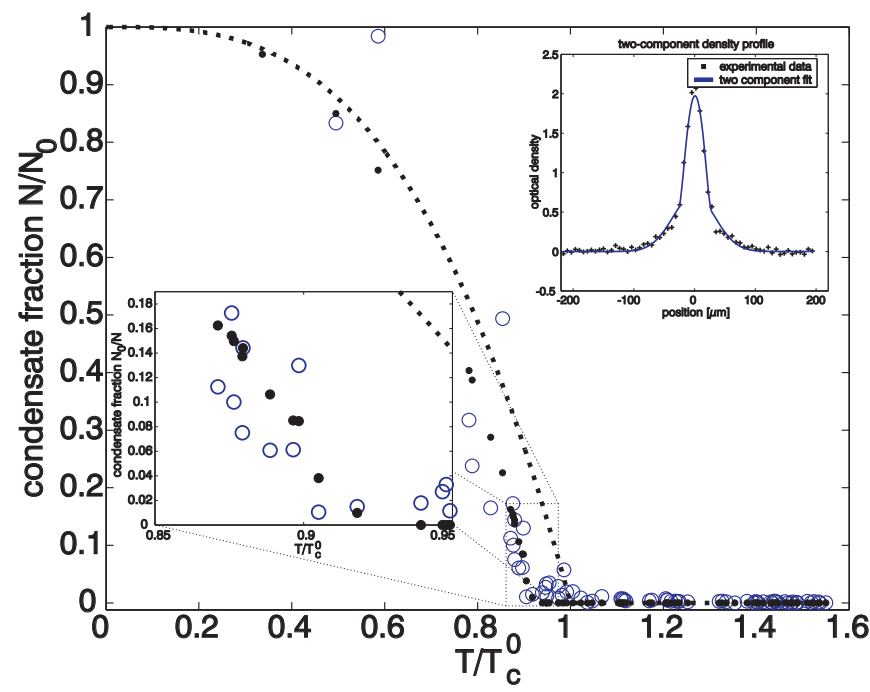

FIG. 2: Condensate fraction $\left(N_{0} / N\right)$ dependence on temperature relative to the transition temperature of an ideal gas $\left(T / T_{C}^{0}\right), T_{C}^{0} \approx 0.94 \frac{\hbar \omega}{k_{B}} N^{1 / 3}$. Open circles represent the measured data. Black dots represent the predicted fraction $\frac{N}{N_{0}}=1-\left(\frac{T}{T_{C}}\right)^{3}$ where $T_{C}=T_{C}^{0}+\delta T_{C}^{i n t}+$ $\delta T_{C}^{f s} . \quad \delta T_{C}^{f s}=-0.73 \frac{\bar{\omega}}{\omega} N^{-1 / 3} T_{C}^{0}$ is a shift in the critical temperature due to the finite number of atoms and $\delta T_{C}^{i n t}=-1.33 \frac{a}{a_{H O}} N^{1 / 6} T_{C}^{0}$ takes into account the contact interaction 32]. Here $a=105 a_{0}$ is the chromium scattering length [24], $a_{0}$ being Bohr's radius, $a_{H O}$ is the harmonic oscillator length, $T$ is the temperature of the thermal cloud, $\omega$ is the geometric and $\bar{\omega}$ the arithmetic mean of the trap frequencies. The dashed curve shows the dependence for the ideal gas.

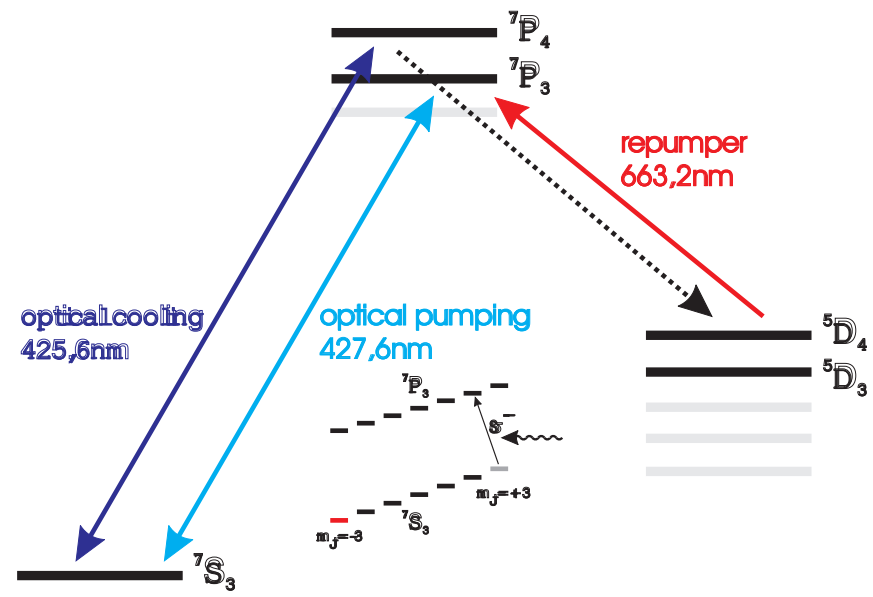

FIG. 3: Chromium levels and lines relevant for cooling and optical pumping.

\section{PRODUCING A CHROMIUM BEC}

The unique electronic structure of chromium (configuration $[\operatorname{Ar}] 3 d^{5} 4 s^{1}$ ) with 6 unpaired electrons with aligned spins in the ${ }^{7} S_{3}$ ground state gives rise to a total spin quantum number of 3 and a very large magnetic moment 


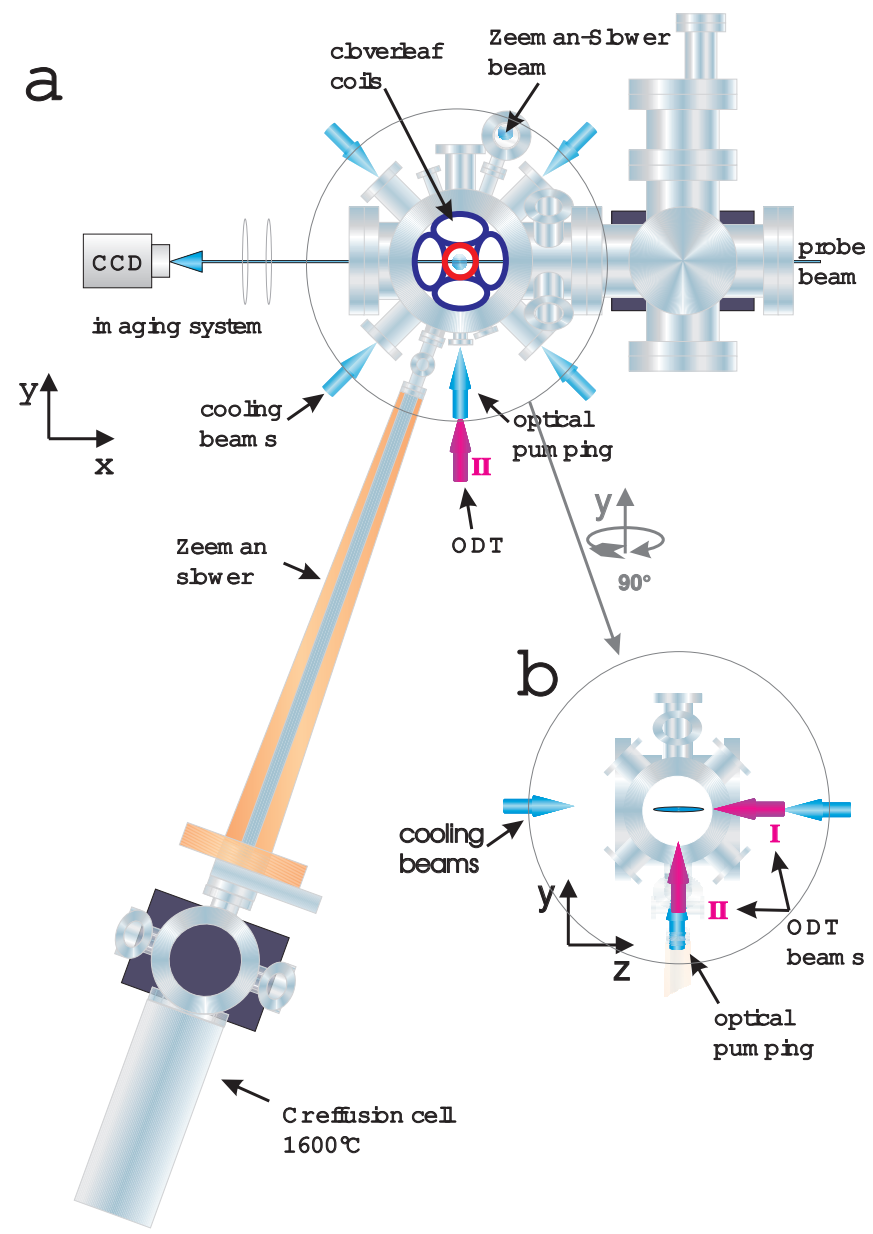

FIG. 4: Schematic setup of our experiment: a) whole apparatus, b) upper chamber seen in the direction of the probe beam. The horizontal beam I of the dipole trap propagates in $\mathrm{z}$-direction and carries a power of up to $9.8 \mathrm{~W}$. The vertical beam II defines our $y$-direction and has a maximum power of $4.8 \mathrm{~W}$.

of $6 \mu_{B}$ making it a well suited element for magnetic trapping. It also has a versatile level-scheme allowing optical cooling and pumping techniques. A drawback however is an extremely large dipolar relaxation rate also arising from the large magnetic moment. In magnetic trapping potentials, trapped atoms are necessarily in the so called low-field seeking states $\left(m_{J}=+3\right)$ and therefore have a high Zeeman energy. Dipolar relaxation processes can lead to a redistribution over the $m_{J}$ levels. The released Zeeman energy leads to atom loss and heating. In earlier experiments [33, 34], these relaxation processes prevented chromium from condensation in a magnetic trap because as the atoms are cooled, the growing spatial density leads to higher and higher two-body loss and heating rates. To circumvent this loss, one has to polarize the atoms in the energetically lowest Zeeman sub state $m_{J}=-3$ where the atoms can not gain any more energy by flipping their spin but would rather have to bring up energy [40]. Therefore dipolar relaxation is suppressed by energy conservation. This state, however, is not magnetically tappable and optical trapping techniques have to be used, where the trapping potential is independent of the Zeeman state [35. The step to an optical dipole trap (ODT) and polarization of the atoms in $m_{J}=-3$ together with an exact alignment of the trapping beams are the essential steps in the preparation process of a chromium BEC which will be described in detail in this section.

Figure 4illustrates schematically the setup which is used for our experiments. The preparation scheme starts with the generation of a beam of chromium atoms by a high temperature effusion cell operating at $1600^{\circ} \mathrm{C}$ and subsequent deceleration by a Zeeman slower. Chromium has a $\Lambda$-like level scheme with weekly allowed intercombination transitions from the excited state of the optical cooling cycle ${ }^{7} S_{3} \leftrightarrow{ }^{7} P_{4}$ at $425.6 \mathrm{~nm}$ into long lived metastable ${ }^{5} D_{3}$ and ${ }^{5} D_{4}$ states (branching ratio $250000: 1$ ). The relevant levels and transitions are shown in fig. 3. Chromium has a high magnetic moment in the ground state as well as in the metastable states. This allows for a continuous loading scheme of optically cooled atoms directly into a magnetic trap with Ioffe-Pritchard (cloverleaf) configuration (CLIP-trap) [36, 37]. With this technique we are able to accumulate about $1.3 \cdot 10^{8}$ atoms within $10 \mathrm{~s}$ at roughly the Doppler temperature of $124 \mu \mathrm{K}$ and a phase space density of a few times $10^{-9}$ in the metastable ${ }^{5} D_{4}$ state. After the steady state number of atoms in the magnetic trap is reached, the cooling lasers are switched off and the atoms in the ${ }^{5} D_{4}$ state are pumped back to the ground state using a diode laser system resonant with the ${ }^{5} D_{4} \leftrightarrow{ }^{7} P_{3}$ transition at $663.2 \mathrm{~nm}$. The magnetic trap is then beeing compressed fully by ramping up currents through the trap coils. In doing so the cloud is heated up again to $\sim 1 \mathrm{mK}$ and Doppler cooling of the optically dense cloud [38] is performed within the trap at an offset field of $14 \mathrm{G}$ using the cooling beams propagating in z-direction. Doppler cooling increases the phase space density by two orders of magnitude without losing atoms. Subsequently, the currents through the coils are lowered to form a trap with an aspect ratio as close as possible to the later shape of the optical dipole trap to provide for efficient transfer between the traps. The offset field in the center of the magnetic trap is adjusted close to $0 \mathrm{G}$ by applying some extra current in the bias coils. In this trap, we perform radio frequency (rf) evaporation with an rf-sweep composed of 3 linear rframps from $45 \mathrm{MHz}$ down to $1.25 \mathrm{MHz}$. After this step, the cloud contains $4 \cdot 10^{6}$ atoms at a phase space density of $10^{-5}$ and a temperature of $22 \mu \mathrm{K}$. The overall gain in phase space density during the magnetic trapping phase is thus about four orders of magnitude while the number of atoms is reduced by 1.5 orders of magnitude.

Beginning from the first step of the rf-ramp, a single beam ODT in horizontal direction with the same symmetry axis as the magnetic trap is shone in. This trap is

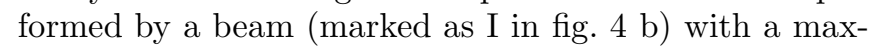
imum of $9.3 \mathrm{~W}$ at a wavelength of $1064 \mathrm{~nm}$ produced by 
a $20 \mathrm{~W}$ Yb-fibre laser (IPG PYL-20M-LP). The beam is focussed to a waist of $30 \mu \mathrm{m}$ in the center of the magnetic trap. The linear polarization of the light allows to split up the light into two beams with adjustable ratio using a polarizing beam splitter and a $\lambda / 2$-plate. The second beam carries up to $4.5 \mathrm{~W}$ and is in a later stage shone in in vertical direction (marked as II in fig. 4) to form a crossed dipole trap with the first beam. Both beam intensities can be controlled independently by acoustooptical modulators.

The final frequency of the rf-sequence is chosen such that the highest number of atoms is remaining in the single beam trap after ramping down the magnetic trapping potential within $100 \mathrm{~ms}$. Perfect alignment of the ODT with best possible overlap between magnetic and optical trap is essential for an efficient transfer into the ODT. In particular, the first beam must be aligned in perfectly horizontal direction because of its rather poor confinement in axial direction resulting in a longitudinal trap frequency of $\sim 13 \mathrm{~Hz}$. In radial direction, the trap frequency is $\sim$ $1450 \mathrm{~Hz}$. Best alignment is achieved by taking absorption images of the dipole trap within the magnetic trap from the side and from above and looking at the alignment of the optical trap with respect to the magnetically trapped cloud. The horizontal beam is walked by using the final two mirrors in the optical path to match direction and position of the beam with the magnetic trap. The longitudinal position of the focus can be varied by moving the final lens which is mounted on a translation stage such that after switching off the magnetic trap, the optical trap stays at the same position.

Immediately after the magnetic trap is off, we pump the atoms to the lowest Zeeman state using about $0.5 \mathrm{~mW}$ of $427.6 \mathrm{~nm}, \sigma^{-}$polarized light and an intensity on the order of $1 \mathrm{~mW} / \mathrm{cm}^{2}$, resonant with the ${ }^{7} S_{3} \leftrightarrow{ }^{7} P_{3}$ transition for which $m_{J}=-3$ is a dark state. The light is produced by a frequency doubled master-slave diode laser system and shone in for $1 \mathrm{~ms}$. To have a well defined alignment of the spins, the pumping process is performed at an offset field of $9 \mathrm{G}$ in y-direction. In contrast to commonly used rf techniques, this optical pumping technique has the advantage that the occupation numbers of the Zeeman substates are not only inverted but the polarization is also purified. This is necessary in our experiment because in the magnetic trap all low-field seeking states are occupied due to dipolar relaxation. The efficiency of the transfer is close to $100 \%$ which is reflected in a dramatic increase of the lifetime of the trapped cloud from $\tau_{+3} \sim 6 \mathrm{~s}$ in $m_{J}=+3$ to $\tau_{-3}>140 \mathrm{~s}$ in $m_{J}=-3$ (see. fig. (5). The magnetic offset field used for pumping is kept on during all further preparation steps to prevent thermal redistribution of the spins.

The transfer efficiency from the magnetic to the optical trap is $40 \%$ and we start with $1.5 \cdot 10^{6}$ atoms in the ODT. They have an initial temperature of $60 \mu \mathrm{K}$ which corresponds to about half the depth of the single beam trap $(130 \mu \mathrm{K})$. The phase space density is $5 \cdot 10^{-5}$, a factor of five higher than the phase space density found when look-

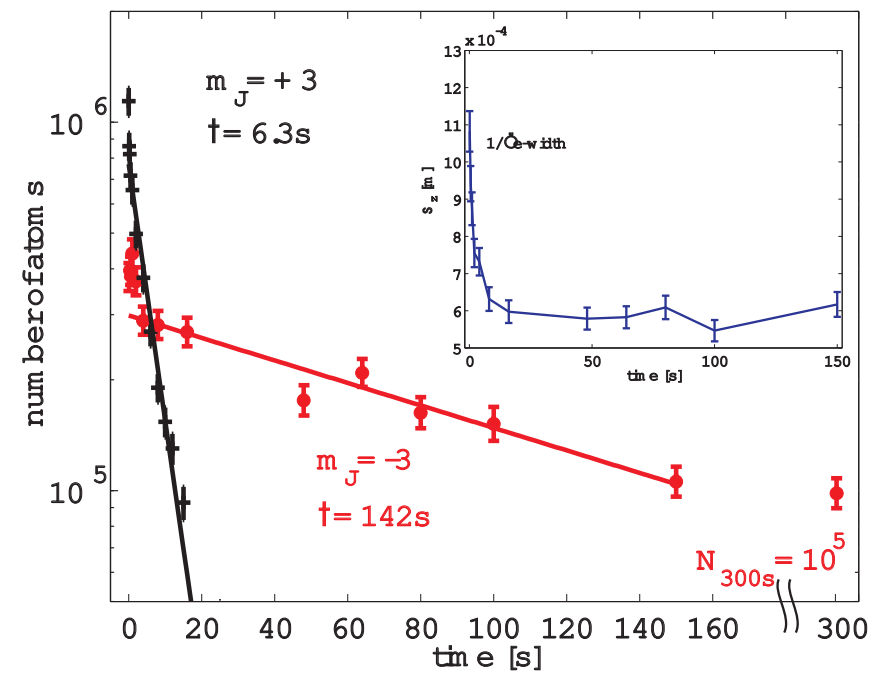

FIG. 5: Comparison of trap lifetimes before (crosses) and after (circles) pumping the atoms to the lowest Zeeman substate. The inset shows the change of the axial size of the expanded cloud in time of flight after different holding times.

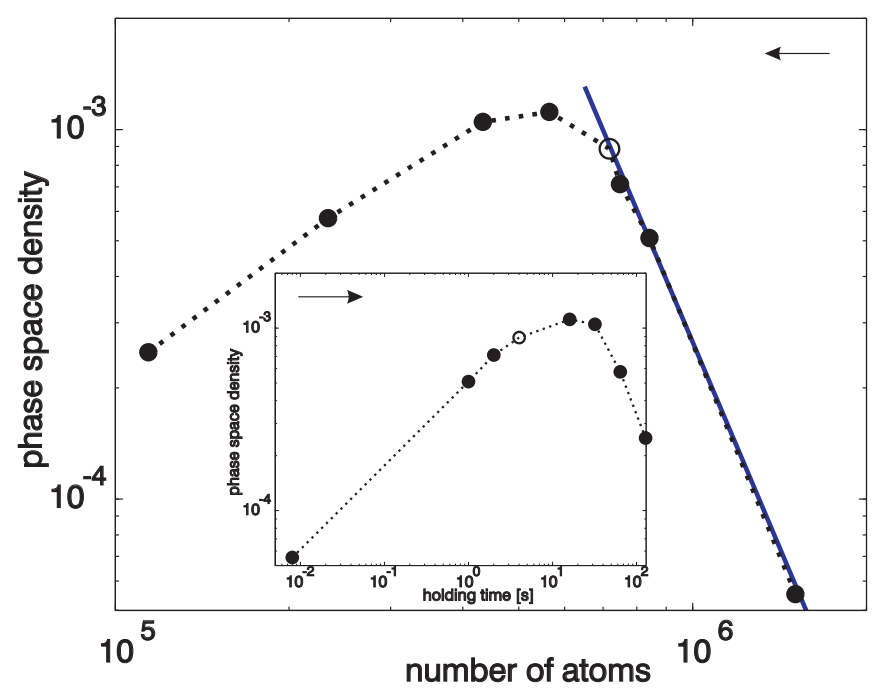

FIG. 6: Double logarithmic plots of the phase space density vs. number of remaining atoms and vs. holding time in the single beam trap (inset). Arrows mark the chronology in the plots. An open circle marks in both plots the optimum point in time to start forced evaporation.

ing at the expansion of the cloud after the final rf-ramp without an optical trap present, although the temperature in the optical trap is significantly higher. During the first seconds in the ODT, a very fast decay is observed which becomes slower after about half of the atoms are lost (fig. 5). Along with this decrease of the number comes a very large increase in the phase space density which was determined from time of flight pictures taken during the lifetime of the trap. We therefore attribute the observed loss to essentially pure plain evaporation. Figure [6] shows the evolution of the phase space density 


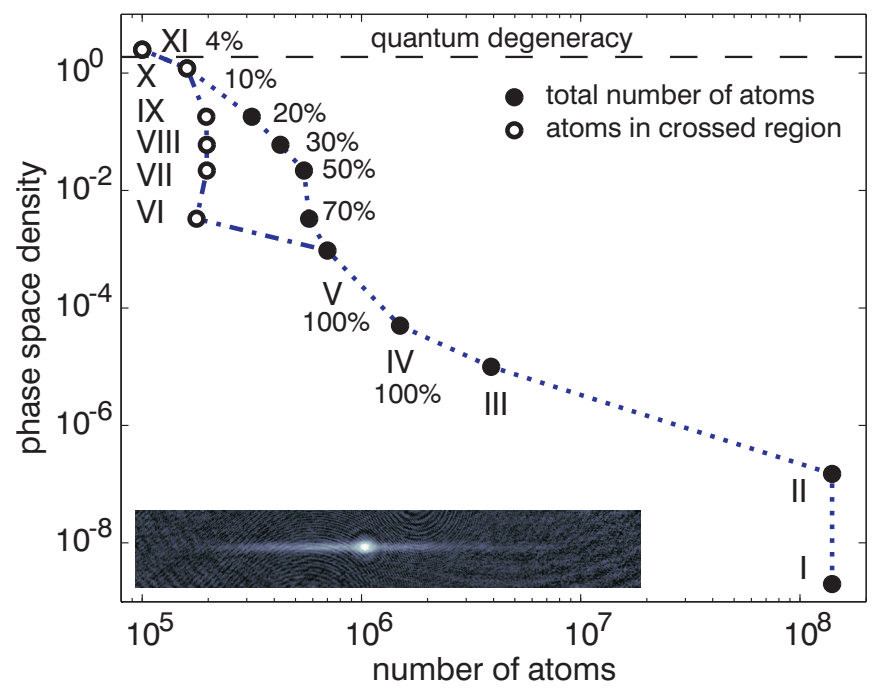

FIG. 7: Evolution of phase space density vs. number of atoms during the whole preparation process starting with 1.3. $10^{8}$ atoms after the CLIP-loading procedure (I). The next steps are Doppler-cooling (II), RF-cooling (III), transfer into the ODT (IV) and initial plain evaporation in the ODT (V). Evaporation is forced from (VI) to (XI). From step (VI) on, filled circles represent the total number of atoms while open circles represent only the atoms trapped in the steep potential in the crossed region. For steps (IV) to (XI), the power in the horizontal trapping beam in percent of the maximum power is also displayed. The vertical beam is ramped up between steps (IV) and (V). The inset shows a typical image of a cloud at $70 \%$ power in the horizontal beam, where a large number of atoms are still located in the wings of the horizontal trapping beam.

during the first $120 \mathrm{~s}$ in the ODT plotted vs. the number of remaining atoms in the main plot and vs. time in the inset. The straight line in the main graph has a slope of 3.6 orders of magnitude gain in phase space density per lost order of magnitude in the number of atoms. This illustrates the very high efficiency of the plain evaporation. We let this evaporation proceed for $5 \mathrm{~s}$ until it gets less efficient which is identifiable by the data points snapping off from the straight line.

During plain evaporation, the second dipole trap beam

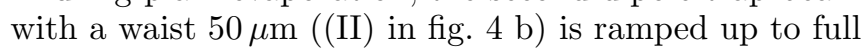
power to form a crossed dipole trap with high trap frequencies in all directions. Best alignment of this beam is achieved by ramping down the first beam, taking an absorption image and improving from shot to shot the number of trapped atoms by adjusting the position of the second beam's focus. Finally, the positioning is adjusted to the number of condensed atoms. In the cooling process, we proceed with forced evaporation in the crossed trap by ramping down the power of the horizontal beam in 6 steps within $5 \mathrm{~s}$ to $70 \%, 1.8 \mathrm{~s}$ to $50 \%, 1.1 \mathrm{~s}$ to $30 \%$, $1.2 \mathrm{~s}$ to $20 \%$, and $1.6 \mathrm{~s}$ to $10 \%$ which is just above condensation. In fig. [7 the phase space densities after each of these evaporation steps are represented by steps (VI) to (XI). From step (VI) on, open circles represent the num- ber of atoms trapped in the crossed region of the trap. Particularly during the first evaporation steps, a larger number of atoms are still trapped in the outer regions ("wings") of the first beam shown in the inset of figure [7] During the evaporation process, atoms are permanently loaded from the wings into the crossed region, causing the number of atoms in this region to stay almost constant during steps (VI) to (IX). After stage (IX) finally all remaining atoms are in the crossed trap and the wings of the horizontal beam are empty. From there on we lose less than half of the atoms in steps (X) and (XI) until quantum degeneracy is reached at a remaining $\sim 8 \%$ of the initial power corresponding to $\sim 800 \mathrm{~mW}$. Large condensates with up to 100.000 atoms are created when we ramp down to $4 \%$ of the maximum power. This last ramp takes $250 \mathrm{~ms}$ and we additionally hold the trap at constant power for another $250 \mathrm{~ms}$ to let the system equilibrate before we release the atoms from the trap to take an absorption image.

The overall gain in phase space density during the optical trapping period starting at $5 \cdot 10^{-5}$ with $1.5 \cdot 10^{6}$ atoms and ending on the order of 1 with $10^{5}$ atoms is more than 4 orders of magnitude, while we lose only 1.2 orders in the number of atoms. Thus the overall quotient of orders of PSD gain vs. orders of atom loss is $>3.6$, almost twice as large as in the magnetic trap. This suggests the use of higher laser power for the optical trap to form a deeper potential and therefore be able to reduce the time of rf-evaporation in the magnetic trap and transfer the atoms into the ODT already at an earlier stage.

\section{TRAP FREQUENCY MEASUREMENT}

Exact knowledge of the trap frequencies is necessary for all measurements where the density distribution of the atoms in the trap is important and where the phase space density of the sample has to be known. In the crossed dipole trap, we measure them independently using a parametric heating technique with a trapped thermal cloud. The preparation of the sample is the same as for the condensation except that the evaporation ramp is stopped before reaching degeneracy. The intensities of the trapping beams are then adiabatically (within $\sim 100 \mathrm{~ms}$ ) changed to form the trapping potential that has to be calibrated. After the cloud is prepared in this way, a remote programmable function generator (Stanford Research Systems DS345) in burst mode is used to modulate the intensity of one of the trapping beams by a few percent $(<5 \%)$ by varying the rf-power of one of the AOMs sinusoidally for $\sim 500 \mathrm{~ms}$. In a series of experiments, the preparation scheme is then repeated, varying from shot to shot the frequency at which the function generator modulates the laser power. After each experiment, an absorption image of the cloud is taken after a time of free ballistic expansion of $4 \mathrm{~ms}$. If the modulation frequency is close to twice the trap frequency in one direction $\omega_{\text {mod }}=2 \omega_{\text {trap }}$ or subharmonics $\omega_{\text {mod }}=2 \omega_{\text {trap }} / n$, 


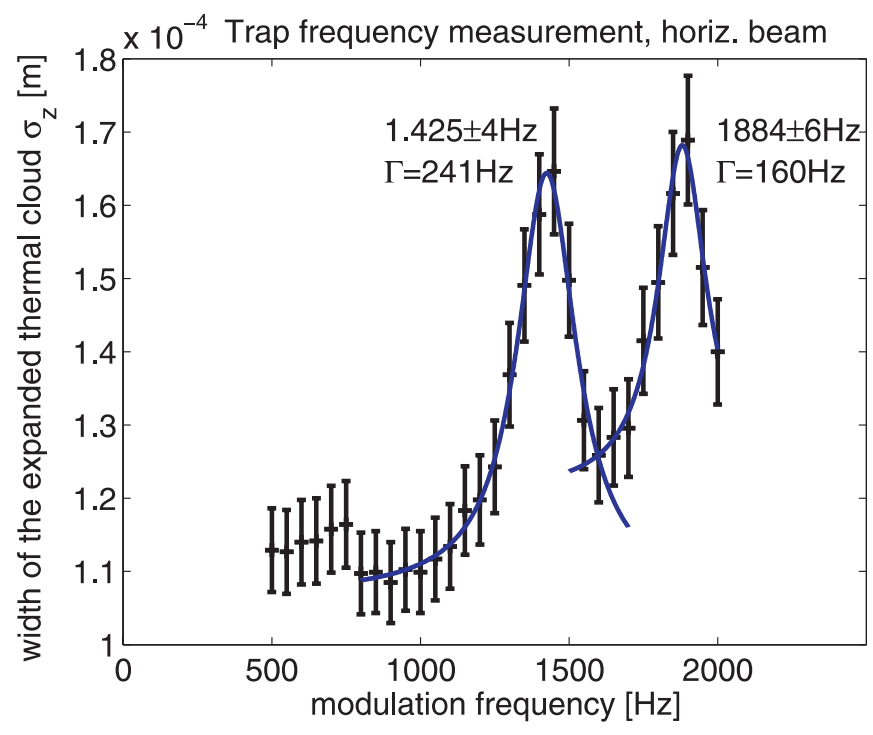

FIG. 8: Measurement of the trap frequencies by modulation of the horizontal beam. $1 / \sqrt{e}$-width of the cloud in z-direction after time of flight plotted over the modulation frequency. The pronounced peaks at $1425 \mathrm{~Hz}$ and $1884 \mathrm{~Hz}$ are at twice the trap frequencies in $\mathrm{y}$ - and $\mathrm{x}$-direction, respectively.

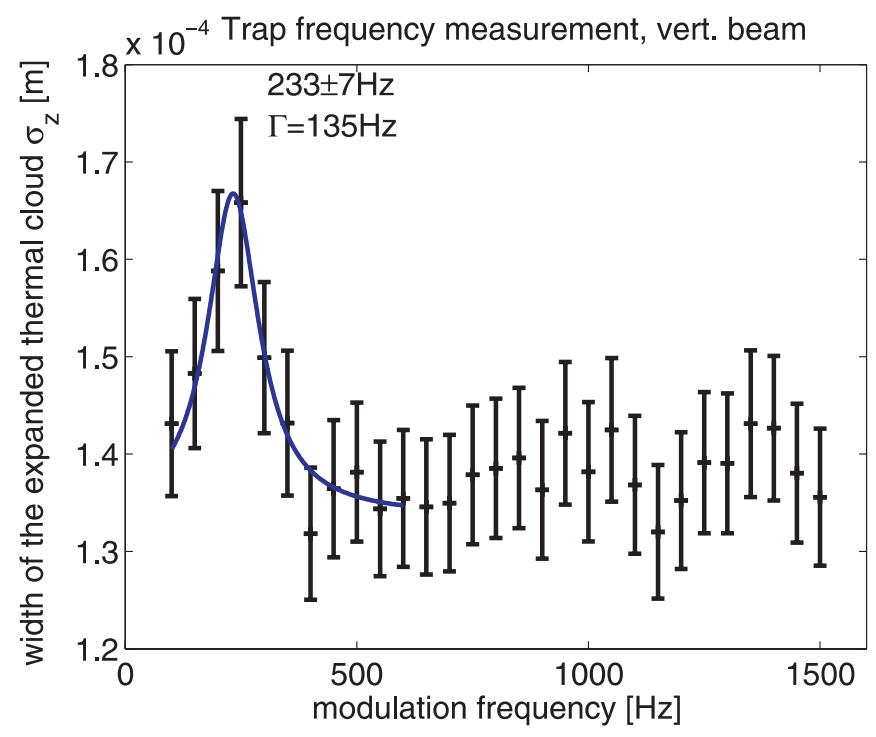

FIG. 9: Measurement of the trap frequencies by modulation of the vertical beam. The resonance peak is at $233 \mathrm{~Hz}$, twice the trap frequency in $\mathrm{z}$-direction. energy is transferred to the atoms and the atomic cloud heats up [35]. This heating effect is most effective at twice the trap frequency which is reflected in a decrease of the number of atoms and a drastic increase of the width of the expanded cloud.

In a crossed dipole trap, each beam mainly contributes to the trap frequencies only in the directions orthogonal to the beam, whereas its contribution in the longitudinal direction is rather small and this frequency is mainly determined by the other beam. Therefore modulating the intensity of one beam allows to resolve only the frequencies in the two orthogonal directions. To measure the trap frequencies in all three directions, the described sequence has to be performed twice, modulating the horizontal beam in one sequence and the vertical beam in the other. Because the temperature and therefore the size of the expanded cloud shows much less shot to shot fluctuations than the number of atoms, we plot the measured widths of the cloud for the whole series over the used modulation frequencies and fit a Lorentz-function to the data around each resonance peak. Examples of such measurements are shown in figures 8 and 9

\section{CONCLUSION}

In conclusion, we have described in detail the improved methods used to generate Bose-Einstein condensates of chromium atoms. With these methods, we are able to produce condensates with $10^{5}$ atoms which is a very good basis for promising further experiments. The preparation makes use of magneto-optical, magnetic and optical techniques which are adapted to the special electronic and magnetic properties of chromium. The evolution of phase space density during the preparation process has been discussed and the methods used to characterize the optical trap have been presented.

\section{Acknowledgments:}

We thank all members of our atom optics group for their encouragement and practical help. We thank Luis Santos, Paolo Pedri, Stefano Giovanazzi, and Andrea Simoni for stimulating discussions. This work was supported by the SPP1116 and SFB/TR21 of the German Science Foundation (DFG).
[1] Axel Griesmaier, Jörg Werner, Sven Hensler, Jürgen Stuhler, and Tilman Pfau. Bose-Einstein condensation of chromium. Phys. Rev. Lett., 94:160401, 2005.

[2] M. H. Anderson, J. R. Ensher, M. R. Matthews, C. E. Wieman, and E. A. Cornell. Observation of Bose-Einstein condensation in a dilute atomic vapor. Science, 269:198, 1995.

[3] K. B. Davis, M.-O. Mewes, M. R. Andrews, N. J. van Druten, D. S. Durfee, D. M. Kurn, and W. Ketterle. Bose-Einstein condensation in a gas of sodium atoms. 
Phys. Rev. Lett., 75:3969, 1995.

[4] C. C. Bradley, C. A. Sackett, J. J. Tollett, and R. G. Hulet. Evidence of Bose-Einstein condensation in an atomic gas with attractive interactions. Phys. Rev. Lett., 75:1687, 1995.

[5] D. G. Fried, T. C. Killian, L. Willmann, D. Landhuis, S. C. Moss, D. Kleppner, and T. J. Greytak. Bose-Einstein condensation of atomic hydrogen. Phys. Rev. Lett., 81:3811, 1998.

[6] G. Modugno, G. Ferrari, G. Roati, R. J. Brecha, A. Simoni, and M. Inguscio. Bose-Einstein condensation of potassium atoms by sympathetic cooling. Science, 294:1320, 2001.

[7] A. Robert, O. Sirjean, A. Browaeys, J. Poupard, S. Nowak, D. Boiron, and C.I. Westbrook A. Aspect. A Bose-Einstein condensate of metastable atoms. Science, 292:461-464, 2001.

[8] Tino Weber, Jens Herbig, Michael Mark, HannsChristoph Nägerl, and Rudolf Grimm. Bose-Einstein Condensation of Cesium. Science, 299:232-235, 2003.

[9] Y. Takasu, K. Maki, K. Komori, T. Takano, K. Honda, M. Kumakura, T. Yabuzaki, and Y. Takahashi. Spin-singlet Bose-Einstein condensation of two-electron atoms. Phys. Rev. Lett., 91(4):040404, 2003.

[10] L. P. Pitaevskii and Sandro Stringari. Bose-Einstein Condensation. Oxford University Press, Oxford, 2003.

[11] M. Inguscio, S. Stringari, and C.E. Wieman, editors. Proceedings of the International School of Physics Enrico Fermi, Course CXL. IOS Press, Amsterdam, 1999.

[12] M. Greiner, O. Mandel, T. Esslinger, T.W. Hänsch, and I. Bloch. Quantum phase transition from a superfluid to a Mott insulator in a gas of ultracold atoms. Nature, 415:39, 2002.

[13] T. Stöferle, H. Moritz, C. Schori, M. Köhl, and T. Esslinger. Transition from a strongly interacting $1 \mathrm{~d}$ superfluid to a Mott insulator. Phys. Rev. Lett., 92:130403, 2004.

[14] B. Paredes, A. Widera, V. Murg, O. Mandel, S. Fölling, I. Cirac, G. V. Shlyapnikov, T. W. Hänsch, and I. Bloch. Tonks-Girardeau gas of ultracold atoms in an optical lattice. Nature, 429:277, 2004.

[15] Stephan Dürr, Thomas Volz, Andreas Marte, and Gerhard Rempe. Observation of molecules produced from a Bose-Einstein condensate. Phys. Rev. Lett., 92(2):020406, 2004.

[16] Jens Herbig, Tobias Kraemer, Michael Mark, Tino Weber, Cheng Chin, Hanns-Christoph Nagerl, and Rudolf Grimm. Preparation of a Pure Molecular Quantum Gas. Science, 301(5639):1510-1513, 2003.

[17] M. Greiner, C. A. Regal, and D.S. Jin. Emergence of a molecular Bose-Einstein condensate from a Fermigas. Nature, 426:537-540, 2003.

[18] M. Bartenstein, A. Altmeyer, S. Riedl, S. Jochim, C. Chin, J. Hecker Denschlag, and R. Grimm. Crossover from a molecular Bose-Einstein condensate to a degenerate Fermi gas. Phys. Rev. Lett., 92:120401, 2004.

[19] M. W. Zwierlein, C. A. Stan, C. H. Schunck, S. M. F. Raupach, A. J. Kerman, and W. Ketterle. Condensation of pairs of fermionic atoms near a Feshbach resonance. Phys. Rev. Lett., 92:120403, 2004.

[20] T. Bourdel, L. Khaykovich, J. Cubizolles, J. Zhang F. Chevy, M. Teichmann, L. Tarruell, S.J.J.M.F. Kokkelmans, and C. Salomon. Experimental study of the BECBCS crossover region in lithium 6. Phys. Rev. Lett.,
93:050401, 2004.

[21] M. W. Zwierlein, J. R. Abo-Shaeer, A. Schirotzek, C. H. Schunck, and W. Ketterle. Vortices and superfluidity in a strongly interacting Fermi gas. Nature, 435:1047-1051, 2005.

[22] S. Giovanazzi, A. Görlitz, and T. Pfau. Ballistic expansion of a dipolar condensate. J. Opt. B: Quantum Semiclass. Opt., 5:S208-S211, 2003.

[23] Jürgen Stuhler, Axel Griesmaier, Tobias Koch, Marco Fattori, Tilman Pfau, Stefano Giovanazzi, Paolo Pedri, and Luis Santos. Observation of dipole-dipole interaction in a degenerate quantum gas. Phys. Rev. Lett., 95:150406, 2005.

[24] J. Werner, A. Griesmaier, S. Hensler, A. Simoni, E. Tiesinga, J. Stuhler, and T. Pfau. Observation of Feshbach resonances in an ultracold gas of ${ }^{52} \mathrm{Cr}$. Phys. Rev. Lett., 94:183201, 2005.

[25] S. Yi, L. You, and H. Pu. Quantum phases of dipolar spinor condensates. Phys. Rev. Lett., 93:040403, 2004.

[26] Duncan H. J. O'Dell, Stefano Giovanazzi, and Claudia Eberlein. Exact hydrodynamics of a trapped dipolar Bose-Einstein condensate. Phys. Rev. Lett., 92:250401, 2004.

[27] K. Góral and L. Santos. Ground state and elementary excitations of single and binary Bose-Einstein condensates of trapped dipolar gases. Phys. Rev. A, 66(2):023613, 2002.

[28] L. Santos, G. V. Shlyapnikov, P. Zoller, and M. Lewenstein. Bose-Einstein condensation in trapped dipolar gases. Phys. Rev. Lett., 85:1791, 2000.

[29] L. Santos, G. V. Shlyapnikov, and M. Lewenstein. Roton-maxon spectrum and stability of trapped dipolar Bose-Einstein condensates. Physical Review Letters, 90(25):250403, 2003.

[30] S. Giovanazzi, A. Görlitz, and T. Pfau. Tuning the dipolar interaction in quantum gases. Phys. Rev. Lett., 89:130401, 2002.

[31] Martin Brinkmann and Jochen Kronjäger. Institut für Laser-Physik, Universität Hamburg, private communication, 2005.

[32] S. Giorgini, L. P. Pitaevskii, and S. Stringari. Condensate fraction and critical temperature of a trapped interacting bose gas. Phys. Rev. A, 54:R4633, 1996.

[33] S. Hensler, J. Werner, A. Griesmaier, P. O. Schmidt, A. Görlitz, T. Pfau, and K. Rzążewski S. Giovanazzi. Dipolar relaxation in an ultra-cold gas of magnetically trapped chromium atoms. Appl. Phys. B, 77:765-772, 2003.

[34] Sven Hensler. Wechselwirkungen in ultrakalten dipolaren Gasen. Phd thesis, 5. Physikalisches Institut, Universität Stuttgart, Pfaffenwaldring 57, 70550 Stuttgart, 2004.

[35] R. Grimm, Matthias Weidemüller, and Yuri B. Ovchinnikov. Optical dipole traps for neutral atoms. Advances in Atomic, Molecular, and Optical Physics, 42:95-170, 2000.

[36] P. O. Schmidt, S. Hensler, J. Werner, Th. Binhammer, A. Görlitz, and T. Pfau. Continuous loading of cold atoms into a ioffe-pritchard magnetic trap. J. Opt. B: Quantum Semiclass. Opt., 5:S170-S177, 2003.

[37] Jürgen Stuhler, Piet O. Schmidt, Sven Hensler, Jörg Werner, Jürgen Mlynek, and Tilman Pfau. Continuous loading of a magnetic trap. Phys. Rev. A, 64:031405(R), 2001.

[38] Piet O. Schmidt, Sven Hensler, Jörg Werner, Thomas 
Binhammer, Axel Görlitz, and Tilman Pfau. Doppler cooling of an optically dense cloud of trapped atoms. J. Opt. Soc. Am. B, 20(5):960-967, 2003.

[39] S. Hensler, A. Greiner, J. Stuhler, and T. Pfau. Depolarisation cooling of an atomic cloud. Europhys. Lett.,
71:918, 2005.

[40] This effect has recently been suggested to be used for a novel cooling scheme [39]. 